\title{
Successful pregnancy following transmyometrial embryo transfer after robot-assisted radical trachelectomy
}

\author{
Hye Jeong Hue ${ }^{1}$, Hyun Ji Choi ${ }^{1}$, Jee Yoon Park ${ }^{1,2}$, Dong Hoon Suh ${ }^{1,2}$, Jung Ryeol Lee ${ }^{1,2}$, Byung Chul Jee ${ }^{1,2}$, Seul Ki Kim ${ }^{1,2}$ \\ 'Department of Obstetrics and Gynecology, Seoul National University Bundang Hospital, Seongnam; ${ }^{2}$ Department of Obstetrics and Gynecology, \\ Seoul National University College of Medicine, Seoul, Korea
}

Radical trachelectomy is a fertility-preserving alternative to radical hysterectomy in carefully selected young women with early-stage cervical cancer. However, in cases with subsequent severe cervical stenosis, assisted reproductive techniques can be difficult. This is a case report of a 34-year-old patient who underwent robot-assisted radical trachelectomy and cerclage for early-stage (IB2) adenosquamous carcinoma. Three months after surgery, the patient underwent ovarian stimulation using a gonadotropin-releasing hormone antagonist protocol. As it was impossible to perform transcervical embryo transfer due to the almost complete absence of the cervical opening, transmyometrial embryo transfer under ultrasound guidance was performed. This resulted in a successful singleton pregnancy. This is the first case of successful pregnancy conceived by in vitro fertilization with transmyometrial embryo transfer in a patient who had previously undergone robot-assisted radical trachelectomy.

Keywords: Embryo transfer; Fertility preservation; Oocyte retrieval; Trachelectomy; Uterine cervical neoplasms

\section{Introduction}

Early detection of cervical cancer increases the chance that fertility-preserving surgery can be performed. However, embryo transfer can be complicated by cervical abnormalities, such as cervical stenosis, atresia, or previous trachelectomy. According to two previous studies, the live birth rate after radical trachelectomy appears to be around $64 \%-67 \%$, and most pregnancies are supported by intrauterine insemination or in vitro fertilization (IVF). Reasons for infertility after trachelectomy include cervical stenosis, decreased cervical

Received: September 3, 2020 • Revised: November 18, 2020 .

Accepted: November 28, 2020

Corresponding author: Seul Ki Kim

Division of Reproductive Endocrinology and Infertility, Department of Obstetrics and Gynecology, Seoul National University Bundang Hospital, 82 Gumi-ro 173beon-gil, Bundang-gu, Seongnam 13620, Korea

Tel: +82-31-787-7264 Fax: +82-31-787-4054 E-mail: drksk80@gmail.com

This is an Open Access article distributed under the terms of the Creative Commons Attribution Non-Commercial License (http://creativecommons.org/licenses/by-nc/4.0/) which permits unrestricted non-commercial use, distribution, and reproduction in any medium, provided the original work is properly cited. mucus, and decreased vasculature supplying the uterus, according to previous studies $[1,2]$. The rate of cervical stenosis due to cervical cancer only or cervical trachelectomy in cases of difficult embryo transfer is challenging to obtain. In about $1 \%$ of cases, experienced experts have found cervical embryo transfer to be difficult due to anatomical and pathological cervical disorders [3]. Doctors may try traumatic transcervical embryo transfer, cervical dilation (with or without hysteroscopy), and transmyometrial embryo transfer (TMET) in such patients.

Kato et al. [4] introduced the Towako method for TMET and achieved several successful cases of pregnancy using this method. The Towako set consists of a needle/stylet and transfer catheter. Under transvaginal ultrasonography (TV-USG) guidance, a needle is inserted transmyometrialy between the upper cervix and lower bladder. When the needle reaches the uterine cavity, it is pulled back until the tip becomes clearly visible at the center of the endometrium. The inner catheter- loaded with one embryo in the culture mediumis then moved through the outer catheter, and the embryo is deposited in the uterine cavity [4]. A retrospective study showed an im- 
plantation rate of $9.5 \%$, with pregnancy and miscarriage rates of $25 \%$ and $30 \%$, respectively, after TMET [3].

There have been no reports of pregnancy with TMET after robotic trachelectomy. Herein, we introduce the first successful case of pregnancy with TMET after robotic trachelectomy.

\section{Case report}

A 34-year-old woman was diagnosed with cervical intraepithelial neoplasia grade 3 with human papillomavirus 16 after a cervical punch biopsy at a local gynecological hospital in December 2019. Soon after, she was transferred to Seoul National University Bundang Hospital for further evaluation. As a result of a loop electrosurgical excision procedure, a 6-mm-deep and 11-mm-wide adenocarcinoma was found. According to magnetic resonance imaging and positron emission tomography, there was no lymph node or distant metastasis. The patient was nulligravida and wanted to become pregnant.

The oncologist classified her disease as stage IB1 (FIGO Committee on Gynecologic Oncology, 2019). Robotic radical trachelectomy, sentinel lymph node mapping, and cervical cerclage were successfully performed in March 2020. The final pathology report confirmed that the adenocarcinoma component was a mixture of the usual type and mucinous carcinoma, and that cancer might remain in the uterus.

The disease classified as stage IB2 $p$ without parametrial or lymphovascular invasion. Therefore, an additional margin was resected and an endometrial biopsy was performed; no tumor was observed. In February and May 2020, it was shown that the squamous cell carcinoma antigen level was $0.6 \mathrm{ng} / \mathrm{mL}$, and the patient decided to postpone additional surgery and get pregnant quickly.

She had been married for 8 years, had a regular menstrual cycle from the age of 15 years, and had no history of other chronic medical or surgical disease; she was a non-smoker and had no history of hirsutism or galactorrhea. Her physical examination was uneventful, except for severe cervical stenosis without a cervical opening. A hormonal work-up showed a normal profile with a day-3 follicle-stimulating hormone (FSH) concentration of $2.05 \mathrm{mlU} / \mathrm{mL}$ and an anti-Müllerian hormone level of $1.52 \mathrm{ng} / \mathrm{mL}$. Hysterosalpingography was normal. Her male partner was 39 years old and had no significant medical history. A semen analysis showed decreased motility (33\%) 5 years ago.

Ovarian stimulation using a gonadotropin-releasing hormone $(\mathrm{GnRH})$ antagonist protocol and IVF/intracytoplasmic sperm injection (ICSI) were selected, considering the patient's age, cervical status, and borderline ovarian reserve. The patient's last menstrual period was June 12 and human FSH (300 IU/day; Gonal-F, Merck Serono, Middlesex, UK) was injected subcutaneously from June 14 to 21. Day
5 TV-USG showed a normal uterus and ovaries. Each ovary had dominant follicles measuring 11-12 mm and a total antral follicle count of eight follicles. Subcutaneous injection of $0.25 \mathrm{mg}$ of $\mathrm{GnRH}$ antagonist (Cetrotide; Serono, Geneva, Switzerland) was performed on day 6 for 4 days. On day 9, TV-USG showed two dominant follicles with mean diameters of $18 \mathrm{~mm}$ or more and an endometrial thickness of $9.7 \mathrm{~mm}$. On the same day, $250 \mu \mathrm{g}$ of recombinant human chorionic gonadotropin (hCG; Ovidrel, Sereno) was administered subcutaneously on the same day to induce ovulation.

The estradiol level on the hCG day was 2,604 pg/mL, and the progesterone level was $0.84 \mathrm{ng} / \mathrm{mL}$. Oocyte retrieval was performed 36 hours after hCG injection under TV-USG guidance. A total of 11 oocytes were retrieved, including three oocytes in metaphase II. ICSI was performed for all available oocytes due to the poor sperm parameters. On day 1, four oocytes showed two pronuclei, which were cleaved into two good-quality embryos (10A and 6A-com). Two embryos were discarded, and one embryo was cryopreserved in a blastocyst (ErB-AB).

As it was impossible to perform transcervical embryo transfer due to the almost complete absence of the cervical opening, TMET under ultrasound guidance was performed using a Towako transfer set (Towako needle; Cook, Eight Mile Plains, Australia) (Figure 1). The pa-
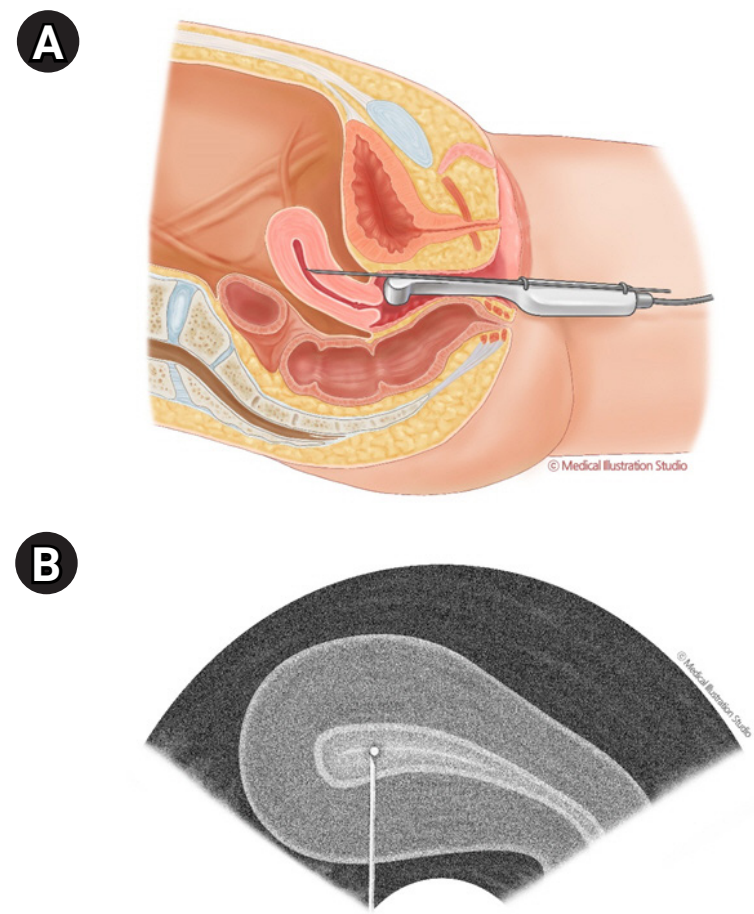

Figure 1. Transmyometrial embryo transfer under ultrasound guidance. (A) Schematic diagram of the procedure: the needle is inserted transmyometrialy and reaches the uterine endometrium. (B) Transvaginal ultrasonography of catheter placement and embryo transfer. 
tient was premedicated with $3 \mathrm{mg}$ of midazolam administered intravenously (IV) and fentanyl (100 $\mu$; IV); both were administered 10 minutes before the procedure. One embryo (EdB-AB) was transferred successfully on the fifth day after oocyte retrieval. There were no major complications, including pain, bleeding, infection, or injuries. Biochemical pregnancy was confirmed 15 days after post-oocyte retrieval by a measurement of serum $\beta$-hCG $(140.83 \mathrm{mlU} / \mathrm{mL})$. An intrauterine fetal heart was confirmed by TV-USG at 7 weeks' gestation.

\section{Discussion}

As early detection of gynecological cancers has become possible, there have been increasingly many patients desiring fertility preservation, particularly in patients with cervical cancer. In cases of stage IB1 cancer and when the tumor $2 \mathrm{~cm}$ or smaller, radical trachelectomy and pelvic lymph node dissection with (or without) para-aortic lymph node dissection may be presented as an option, and abdominal trachelectomy can be performed even if the tumor is $2 \mathrm{~cm}$ or larger [5]. After trachelectomy, cervical dilation is difficult, and it is difficult to reach the fundus.

Our patient underwent robot-assisted radical trachelectomy with clinical-stage IB1 disease, but the final pathology report confirmed that the adenocarcinoma component was a mixture of the usual type and mucinous carcinoma, and it was found that cancer might remain in the uterus. Thus, additional resection margins and an endometrial biopsy were conducted, with no residual cancer as a result. Because the patient prioritized pregnancy, the consideration of any additional surgery was postponed.

Unlike other cases reported previously, the patient presented herein prioritized pregnancy and attempted to become pregnant immediately after surgery without proper follow-up to determine whether there was recurrence. In our case, for a fast pregnancy, TMET was performed immediately after trachelectomy, and a successful pregnancy was achieved.

With the development of assisted reproductive technology technology, it is important to increase the implantation and pregnancy rates through appropriate embryo transfer. According to a retrospective observational study, it was estimated that a poor embryo transfer technique accounted for 30\% of all IVF failures. Embryo transfer was difficult in about $7.7 \%$ of all procedures, and the clinical pregnancy rate (CPR) was significantly lower than in non-difficult procedures (27.1\% vs. $38.2 \%, p<0.001)$. The CPR decreased progressively with the use of additional maneuvers during embryo transfer, including the use of an outer catheter sheath, Wallace stylet, and tenacula [6].

If there are cervical abnormalities, such as cervical stenosis, atresia, or previous trachelectomy, the likelihood of needing an additional maneuver increases. In such cases, TMET can be considered. Accord- ing to Khairy et al. [7], when performing TMET, endometrial trauma and myometrial contraction can be reduced by minimizing the procedure time, and the risk of contamination of the embryo catheter and infection can be reduced by not passing through cervical secretions.

In a previous report, a patient had cervical cancer (1A2) and attempted a natural pregnancy for 6 months after radical vaginal trachelectomy and laparoscopic pelvic lymph node dissection 3 years previously; however, she was unsuccessful. As a result, she tried IVF using a GnRH agonist long protocol with TMET and was presented as a case of successful pregnancy [8]. Another patient had a distorted internal cervical canal and cervical dilation failed repeatedly. She also achieved pregnancy by TMET [9].

Khairy et al. [7] compared the outcomes of "very difficult transcervical embryo transfer" (vdTCET) versus TMET in 174 patients. They defined vdTCET as requiring more than two tenacula or stylets, changing the embryo transfer catheter, reloading of the embryos or canceling the procedure, and freezing the embryo to transfer after cervical dilatation. The clinical pregnancy and live birth rates for TMET (32.6\% and 25\%, respectively) were higher than those for vdTECT (26.1\% and $16.4 \%$, respectively). These studies have shown that TMET is a good alternative for difficult embryo transfer cases where cervical embryo transfer cannot be achieved.

During the follow-up period, it is necessary to check for miscarriage, live birth, or recurrence of cervical cancer. This is the first case of successful pregnancy conceived by IVF with TMET for a patient who had previously undergone robot-assisted radical trachelectomy. In the future, it may be meaningful to consider providing the options of ART and TMET in women of childbearing age diagnosed with cervical cancer when additional surgery is needed after a quick pregnancy.

\section{Conflict of interest}

Byung Chul Jee is an Editor-in-Chief and Seul Ki Kim is an Associate Editor of the journal, but they were not involved in the peer reviewer selection, evaluation, or decision process of this article. No other potential conflicts of interest relevant to this article were reported.

\section{Acknowledgments}

We are deeply appreciative of Medical Art Studio (Sun Joo Kim, Suhyun Chea) for a medical illustration visualizing our concept.

\section{ORCID}

Hye Jeong Hue https://orcid.org/0000-0002-8409-3157 


$\begin{array}{ll}\text { Hyun Ji Choi } & \text { https://orcid.org/0000-0002-4161-0708 } \\ \text { Jee Yoon Park } & \text { https://orcid.org/0000-0002-2047-5703 } \\ \text { Dong Hoon Suh } & \text { https://orcid.org/0000-0002-4312-966X } \\ \text { Jung Ryeol Lee } & \text { https://orcid.org/0000-0003-3743-2934 } \\ \text { Byung Chul Jee } & \text { https://orcid.org/0000-0003-2289-6090 } \\ \text { Seul Ki Kim } & \text { https://orcid.org/0000-0002-1647-6711 }\end{array}$

\section{Author contributions}

Conceptualization: SKK. Data curation: HJH. Formal analysis: $\mathrm{HJH}$, SKK. Project administration: SKK, JYP, DHS, JRL, BCJ, HJC. Writingoriginal draft: $\mathrm{HJH}$. Writing-review \& editing: $\mathrm{HJH}, \mathrm{SKK}$.

\section{References}

1. Willows $K$, Lennox G, Covens A. Fertility-sparing management in cervical cancer: balancing oncologic outcomes with reproductive success. Gynecol Oncol Res Pract 2016;3:9.

2. Bentivegna E, Maulard A, Pautier P, Chargari C, Gouy S, Morice P. Fertility results and pregnancy outcomes after conservative treatment of cervical cancer: a systematic review of the literature. Fertil Steril 2016;106:1195-211.e5.

3. Ferreri J, Portillo EG, Penarrubia J, Vidal E, Fabregues F. Transmyometrial embryo transfer as a useful method to overcome difficult embryo transfers: a single-center retrospective study. JBRA Assist Reprod 2018;22:134-8.

4. Kato O, Takatsuka R, Asch RH. Transvaginal-transmyometrial embryo transfer: the Towako method; experiences of 104 cases. Fertil Steril 1993;59:51-3.

5. Koh WJ, Abu-Rustum NR, Bean S, Bradley K, Campos SM, Cho KR, et al. Cervical cancer, version 3.2019, NCCN Clinical Practice Guidelines in Oncology. J Natl Compr Canc Netw 2019;17:64-84.

6. Kava-Braverman A, Martinez F, Rodriguez I, Alvarez M, Barri PN, Coroleu B. What is a difficult transfer? Analysis of 7,714 embryo transfers: the impact of maneuvers during embryo transfers on pregnancy rate and a proposal of objective assessment. Fertil Steril 2017;107:657-63.e1.

7. Khairy M, Shah H, Rajkhowa M. Transmyometrial versus very difficult transcervical embryo transfer: efficacy and safety. Reprod Biomed Online 2016;32:513-7.

8. Jamal W, Phillips SJ, Hemmings R, Lapensee L, Couturier B, Bissonnette $F$, et al. Successful pregnancy following novel IVF protocol and transmyometrial embryo transfer after radical vaginal trachelectomy. Reprod Biomed Online 2009;18:700-3.

9. Sullivan-Pyke CS, Kort DH, Sauer MV, Douglas NC. Successful pregnancy following assisted reproduction and transmyometrial embryo transfer in a patient with anatomical distortion of the cervical canal. Syst Biol Reprod Med 2014;60:234-8. 